\title{
¿E1 fin de la hospitalidad? \\ Los conceptos sociales fundamentales de la hospitalidad
}

Leandro Benedini Brusadin ${ }^{1}$

Resumen: La Sociología y la Filosofía se han convertido en una de las premisas fundamentales para la investigación en hospitalidad. El objetivo principal de este artículo es la internacionalización del campo de la hospitalidad, en particular con el enfoque de la Escuela Francesa de pensamiento humano. La metodología se basa en un debate epistemológico sobre los conceptos fundamentales de la hospitalidad pública en el contexto migratorio contemporáneo. La obligación de acoger al otro como una acción de cosmopolitismo moral y una actitud altruista zozobra en la contemporaneidad. La llamada crisis migratoria de nuestro tiempo es, en tales circunstancias, una crisis de hospitalidad.

Palabras clave: hospitalidad pública; conceptos; ciudad; inmigrantes; crisis de la hospitalidad.

\section{O fim da hospitalidade? \\ Os conceitos sociais fundamentais de hospitalidade}

Resumo: A Sociologia e a Filosofia tornaram-se uma das premissas fundamentais para a pesquisa em hospitalidade. O principal objetivo deste artigo é a internacionalização do campo da hospitalidade, em particular com a abordagem da Escola Francesa de pensamento humano. A metodologia baseia-se em um debate epistemológico sobre os conceitos fundamentais de hospitalidade pública no contexto migratório contemporâneo. A obrigação de acolher o outro como uma ação do cosmopolitismo moral e uma atitude altruísta naufragou na contemporaneidade. A chamada crise migratória do nosso tempo é, nessas circunstâncias, uma crise de hospitalidade.

Palavras-chave: hospitalidade pública; conceitos; cidade; imigrantes; crise de hospitalidade.

The end of hospitality?

The fundamental social concepts of hospitality

Abstract: Sociology and Philosophy have become one of the fundamental premises for research in hospitality. The main objective of this paper is the internationalization of the field of hospitality, in particular with the approach of the French School of human thought. The methodology is based on an epistemological debate on the fundamental concepts of public hospitality in the contemporary migratory context. The obligation to host the other as an action of moral cosmopolitanism and an altruistic attitude is shipwrecked in contemporaneity. The so-called migration crisis of our time is, in such circumstances, a crisis of hospitality.

Keywords: public hospitality; concepts; city; immigrants; hospitality crisis.

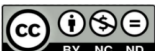

DOI: https://doi.org/10.26512/patryter.v4i7.29654

Como citar este artigo: Brusadin, L. B. (2021). ¿El fin de la hospitalidad? Los conceptos sociales fundamentales de la hospitalidad. PatryTer - Revista Latinoamericana e Caribenha de Geografia e Humanidades, 4 (7), 107-119. DOI: https://doi.org/10.26512/patryter.v4i7.29654

Recebido: 18 de fevereiro de 2020. Aceite: 14 de maio de 2020. Publicado: 01 de março de 2021.

${ }^{1}$ Professor do Departamento de Turismo e do Programa de Pós-Graduação em Turismo e Patrimônio da Universidade Federal de Ouro Preto (UFOP). Professor do Programa de Pós-Graduação em Ambiente Construído e Patrimônio Sustentável da Universidade Federal de Minas Gerais (UFMG). ORCID: https://orcid.org/0000-00022778-2095 E-mail: leandro@ufop.edu.br. 


\section{Introducción}

En 2015, el presente investigador completó su primer postdoctorado en la Escuela de Artes, Ciencias y Humanidades de la Universidad de São Paulo (USP), donde estudió la recepción de viajeros extranjeros naturalistas para el siglo XIX en Minas Gerais (Brasil) y realizó entrevistas con investigadores líderes en el campo de la hospitalidad, incluida la Dra. Anne Gotman, durante nuestra primera reunión cara a cara.

A pesar de la categorización y el análisis de los significados sociales e históricos de los objetos de reinvestigación a partir de estudios realizados anteriormente, que generaron docenas de publicaciones en anuarios de conferencias, revistas y libros, este investigador a menudo se enfrentó a un trabajo insuficientemente más denso en cuanto a hospitalidad e interfaz social. Fue entonces cuando se eligió el tema de esta nueva investigación, porque lo vimos como una oportunidad para el desarrollo científico de la hospitalidad en la comunidad académica brasileña. En consecuencia, el trabajo de investigación diario se ha convertido en un desafío natural para el trabajo académico posterior de este investigador en el proceso de nuestra internacionalización, como la Escuela Francesa de Pensamiento Humano.

Este artículo, fruto de un postdoctorado realizado entre el 1 de septiembre de 2018 y el 31 de agosto de 2019 en la Universidad de París V Descartes (Facultad de Ciencias Humanas y Sociales - Sorbona), en el Centro de Investigación en Enlaces Sociales (Cerlis), bajo la dirección de la doctora Anne Gotman, va exactamente en esta dirección, porque tanto el laboratorio como la directora de este trabajo son referencias académicas para el estudio de las relaciones sociales. Permitieron a este investigador una profundización científica significativa sobre el tema de la hospitalidad y la migración. Las experiencias científicas acumuladas durante este período han proporcionado una maduración intelectual que se afirma que demuestra. Entre estas experiencias, logramos consultar obras inaccesibles en Brasil gracias al acceso a las bibliotecas de la Universidad de París.

A pesar de esto, entendemos que este tipo de estudio profundiza las discusiones que a veces se pasan por alto en algunos campos del pensamiento humano o incluso se usan sólo como fuente superficial simbólica por una supuesta cientificidad del campo, esencialmente con respecto a varias investigaciones sobre hospitalidad realizadas en Brasil, donde predomina el enfoque anglosajón. La hospitalidad tiene, en su matriz de estudio, la premisa del don y la fenomenología de la recepción, que puede convertirse en una corriente de pensamiento referenciada por otros campos científicos en nuestro país.

El hecho es que Mauss y Derrida influyeron en el trabajo de las nuevas generaciones de autores franceses, incluidos Alain Caillé, René Schérez, Hervé Le Bras, Michelle Perrot, Anne Gotman, Jacques T. Godbout, Claude Raffestin, Danielle Perrot y el joven Benjamin Boudou, entre otros. Entre los autores contemporáneos más notables se encuentran Alain Montandon y Anne Gotman (Spolon \& Brusadin, 2016). Este tipo de estudio filosófico y sociológico es de suma importancia porque "ainsi se pose une question, à première vue étrange, qui cherche à savoir s'il existe une relation entre le don de la vie, l'art de la conservation, l'amour filial ou patriotique, le goût du travail bien fait, l'esprit d'équipe, le don de sang et les déjeuners d'affaires" (Godbout, 1992, p. 22). El don debe entenderse como un sistema, este sistema no es más que el sistema social en sí.

Desde esta perspectiva, la hospitalidad no sólo es gratuita, sino que debe ser generosa: para algunos, es un sistema de donación desequilibrado; para otros, tiene su virtud de reequilibrio. La verdadera hospitalidad reside supuestamente en un sistema abierto, capaz de absorber el exceso de personas en cualquier momento, incluso gracias a su contribución. La verdadera hospitalidad (hospitalidad generosa) significa tener que sacrificar espacio y compartirlo con el anfitrión. La hospitalidad generosa es finalmente lo que se dirige a las personas difíciles de aceptar. Cabe señalar que la generosidad no es la ausencia de gratificación, por el contrario. La generosidad es codiciosa y requiere que el don no fluya de una manera. La hospitalidad es un proceso de agregación del anfitrión a la comunidad y la hospitalidad, el proceso inverso, requiere protocolos igualmente estudiados.

\footnotetext{
L’hospitalité est une idée nécessaire et juste qui pose un modèle de l'accueil que l'on doit avoir en vue pour tendre à sa réalisation. Elle est une idée nécessaire car la société repose sur l'hospitalité. Que serait une école qui n'ouvrirait pas ses portes le matin, lorsque les écoliers arrivent? Que seraient les commerces qui refuseraient de laisser entrer des inconnus sous prétexte qu'ils ne se sont pas fait connaître (Brugere \& Le Blanc, 2017, p. 193).
} 
La relación del don es creada por los actos de dar, recibir y devolver a los demás. Si alguien nos hace un favor, por ejemplo, establece una relación a través de la cual probablemente nos sentiremos obligados a hacerle otro favor a cambio (Mauss, 2008). Estas relaciones son sociológicamente importantes porque contribuyen a la cohesión social. El sentido de comunidad se ve así reforzado por la tendencia de las personas a ayudarse mutuamente. De manera similar, la solidaridad familiar y vecinal se basa en cierta medida en este complejo ritual. En las sociedades anteriores a la comercialización, donde los pagos en efectivo desempeñaban un papel insignificante, el intercambio social se basaba en gran medida en los cambios en la proporción de dones asimétricos, aunque inherentes a la lógica del poder.

El territorio es uno de los lugares del don, ya que es el núcleo indiscutible de la vida de las relaciones humanas con sus condiciones particulares de reproducción social y guardián de los significados existenciales. Desde los estilos de vida hasta la relación total de la sociedad que constituye el significado de la humanidad, todos revelan los personajes que producen el territorio a través de usos y apropiaciones; es decir que la apropiación territorial acusa las dimensiones reales de dar, recibir y devolver. El territorio ordenado es la expresión de la vida, de la manera más diversa, intenciones e ideologías (Costa \& Moesch, 2017).

Por esta razón, el sistema de dones de Mauss (2008) integrado en la tríada dar, recibir y devolver puede permitir una mejor comprensión de los fenómenos relacionados, como la asociación, el liderazgo y la solidaridad humana. Aunque el estudio de la hospitalidad no puede ignorar el dominio comercial, sabemos que este último es inherente al sistema capitalista y que no responde a las premisas del don y las aspiraciones incondicionales hacia el otro. En nuestra opinión, es esencial pensar en las virtudes de la hospitalidad con todos los desafíos que implica dicha directiva, ya que los excluidos del sistema capitalista son incapaces de insertarse como protagonistas de sus propias historias.

De esta manera, nuestra investigación ha ponderado la relevancia de las relaciones, intereses, roles y condiciones sociales del don con una justificación epistémica, sabiendo que la teoría de Marcel Mauss proporciona las bases constitutivas de la hospitalidad y que estas limitaciones teóricas son entrelazados para el análisis del caso de los inmigrantes. Sin embargo, Mauss (2008) advirtió que las aplicaciones no pueden confundirse con la ciencia misma. No obstante, surgen numerosas propuestas científicas generalizadoras, sin estar vinculadas a la dinámica social; deben ser reevaluados por el homo academicus. El hecho es que debe establecerse un verdadero debate intelectual con la definición de los límites y posibilidades de cada esfera de conocimiento para el desarrollo científico del campo.

¿Por qué, entonces, un término tan marcado en la historia por el estigma de la nostalgia y la ingenuidad se ha convertido hoy en el tema de algunos estudios científicos? Camargo (2004) indica que esto nos inserta en los problemas sociales inherentes a los males de la globalización, dado que la migración humana continúa y que todavía existe preocupación por la homogeneización gradual de hábitos y costumbres.

Por lo tanto, el objetivo principal de esta investigación es la internacionalización del campo de la hospitalidad, en particular con el enfoque de la Escuela Francesa de pensamiento humano. En este caso, informamos las preguntas de Anne Gotman (1997, p. 14) cuando dijo: "hospitalité, accueil: les logiques ne sont pas équivalentes. L'hospitalité est-elle réductible au supplément de civilité que comporte aujourd'hui l'accueil professionnalisé d'une relation de service accomplie ? Et si l'accueil est une mimésis de l'hospitalité, que mime-t-il au juste ?" El artículo concluye con las principales consideraciones sobre el debate teórico y la investigación sobre las relaciones de hospitalidad con los inmigrantes en la era moderna.

\section{Hospitalidad y hostilidad hacia los inmigrantes en la modernidad}

Las distinciones entre "nosotros" y "otros" son factores clave para comprender las escenas de la hospitalidad pública. Las alteridades entre los protagonistas denotan los procesos de acogida y hostilidad entre quienes son miembros y no miembros de una sociedad determinada. Estas distinciones se postulan en el tejido social contemporáneo entre los nacionales de un país determinado y los extranjeros que afirman compartir este mismo territorio. En esta lógica, surgen algunas preguntas que suenan naturalmente para los miembros que tendrían la función de anfitrión: ¿cuál es el punto de dar una buena bienvenida al extranjero (inmigrante) si se supone que lo que tiene que ofrecer me interesa? Observamos en este comportamiento la ausencia 
total de desinterés y la falta de aspiraciones incondicionales hacia los demás, factores esenciales de hospitalidad en nuestro entendimiento. A esto se suma la xenofobia contra el migrante frente al miedo a perder algo de sí mismo, debido a la interacción con la cultura de los demás.

Lo opuesto a la hospitalidad es la hospitalidad misma, que se convierte en hostilidad u odio. Las prácticas de hospitalidad están aumentando en todos los países involucrados, llevadas por personas anónimas que dan sin contar y apoyan a los exiliados sin exigir nada a cambio (Brugere \& Le Blanc, 2017). Sin embargo, según Gotman (2001, p. 342), "hospitalité et inhospitalité ne sont d'ailleurs pas nécessairement antinomiques, et les oscillations de l'une à l'autre s'observent simultanément dans des régions différentes de l'action sociale".

El hecho es que el estudio de la (crisis de) solidaridad en la modernidad pertenece a la sociología, porque, frente a su carácter abstracto y en la medida en que constituye una simple predisposición de nuestra naturaleza psíquica, la solidaridad es demasiado indefinida para que podamos alcanzarlo fácilmente (Durkheim, 1960). Entonces, pensar por conceptos no es simplemente ver la realidad en el lado más general: es proyectar sobre la sensación una luz que la ilumina, la penetra y la transforma en filosofía de la relación de uno con el otro.

La cuestión de la hospitalidad comienza allí para Derrida y Dufourmantelle (1997): ¿deberíamos pedirle al extranjero que nos entienda, que hable nuestro idioma antes y para poder darle la bienvenida en casa? En la misma línea, Gotman (2004, p. 3) pregunta:

Qui accueille qui, et selon quels critères sont identifiés
les accueillis ? Quelle place la communauté installée des
ressortissants et des résidents ménage à l'étranger,
l'arrivant, le non-ressortissant? Où commence l'autre?
En vertu de quelle légitimité est-il défini ainsi? Quel
régime lui applique-t-on? Quelles sont les
contradictions et les conséquences de cette division entre
ressortissants et non-ressortissants consubstantielle à la
pensée d'État?

En un mundo donde la solidaridad, la compasión, el intercambio, la ayuda mutua y la simpatía (nociones extrañas para el pensamiento económico, y odiadas por la práctica económica) suspenden o descartan la elección racional y la búsqueda del interés propio; los sustitutos comerciales no pueden reemplazar los lazos humanos. En su versión comercial, los enlaces se convierten en bienes y no satisfacen la necesidad de interacción social. Por su parte, la solidaridad humana es la primera en caer por el triunfo del mercado de consumo (Bauman, 2004).

Para Montandon (2002), al considerar este razonamiento, muchos sujetos dejan de pensar que el proceso de bienvenida reactiva la nostalgia primera y arcaica: la seguridad, el calor del refugio, la alimentación continua, el rostro de la madre. Caillé (2002) afirma que la acción del don en la hospitalidad consiste en cualquier prestación de servicios o bienes realizados sin garantía de devolución, con el fin de crear, mantener o regenerar el vínculo social. Sin embargo, la sociedad de individuos está marcada por el predominio de intercambios simétricos y extremadamente interesados, inherentes a la lógica capitalista.

Por tanto, sería difícil para estos argumentos convencer a un Estado o a un miembro que se opone a la presencia de un no miembro a cambiar su opinión, en contra del acto de hacer que la hospitalidad sea adecuada para el ejercicio del derecho humano a la vivienda, salud y trabajo. El hecho es que los gestos de hospitalidad de una sociedad dada hablan mucho de esta última ante las oportunidades y la necesidad de intercambios entre el anfitrión y sus invitados:

\section{Ce protocole d'hospitalité est en effet en tout premier lieu une méthode d'observation de l'arrivant, initiée par les locaux et destinée à jauger ses qualités; une mise à l'épreuve de ses intentions, pacifiques ou guerrières, un examen de passage dont le résultat décidera de son sort, c'est-à-dire de sa transformation ou pas en hôte, puis de son intégration dans la communauté locale et, finalement, des possibilités d'échange entre protagonistes (Gotman, 2013, p. 3).}

$\mathrm{Al}$ recibir a alguien en su hogar (o en su país), se asegura un gesto inherente a la dinámica de dar-recibir-devolver sea precedido o no por una invitación. La hospitalidad asume su función de sedimentarse en el tejido social y, por lo tanto, se convierte en un hecho social insertado en una lógica de intercambio de dones impuesta por la asimetría de las relaciones con una propuesta de aspiración incondicional para el bienestar del otro.

La ley cosmopolita debe limitarse a las condiciones de la hospitalidad universal, pero la hospitalidad kantiana, concebida como un derecho de visita y no de recepción, está muy alejada de la antigua filia y es más extensa. Jacques Derrida distingue entre hospitalidad absoluta e 
incondicional y la ley o pacto de hospitalidad. Por el contrario, nos parece que para ampliar la distancia entre una forma de hospitalidad que ordena abrir el hogar a un "otro absoluto" desconocido y anónimo sin solicitud de reciprocidad, y otro enmarcado por los derechos y deberes de la reciprocidad ofrecida a un extranjero "representado y protegido" por su apellido, perdimos su punto, que es precisamente el juego en el límite entre interés y sacrificio (Gotman, 2001).

De hecho, la paz perpetua de Kant (2006) sigue siendo imposible, pero los medios concretos para lograrlo son accesibles; con todo, esta condición de hospitalidad universal es limitada porque el extranjero no puede tener un derecho de recepción o un derecho de residencia, sino sólo un derecho de visita al territorio de otro. Según Boudou (2017), la hospitalidad kantiana es el principio que hace que el proverbio estadounidense sea ilegal shoot first and ask questions afterwards.

En este contexto, Derrida encontró en Kant el deber de decirle la verdad al otro como un deber absoluto de respeto por él otro y respeto por el vínculo social. Gotman (2017, p. 6) se pregunta: "comment ne pas penser au projet de Paix perpétuelle de Kant qui défendait la nécessité d'une hospitalité universelle dictée par les besoins du commerce, la rondeur et la finitude de la planète et le fait que tous sont inéluctablement conduits à se rencontrer ?". Es "une philosophie politique que Kant se réclame lorsqu'il réduit l'hospitalité à un droit de visite cependant universel, imposé par la nécessité économique de commercer avec le monde et pragmatique de se rencontrer sur une terre à la fois ronde et limitée" (Gotman, 1997, p. 10).

Si comparamos esto con la conducta inhospitalaria de los Estados civilizados y especialmente de los Estados comerciales en nuestra parte del mundo, la injusticia que demuestran hasta el horror cuando visitan países y pueblos extranjeros:

l'idée d'un droit cosmopolitique n'est pas un mode de représentation fantaisiste et extravagant du droit, mais c'est un complément nécessaire du code non écrit, aussi bien du droit public des hommes en général et ainsi de la paix perpétuelle dont on ne peut se flatter de se rapprocher continuellement qu'à cette condition (Kant, 2006, p. 97).
De esta manera, Proust presenta el trabajo de Kant y dice que la paz perpetua no es, por lo tanto, la ausencia de conflicto y el fin de las hostilidades. Esta paz se opone a la guerra, pero no a disputas y conflictos por los cuales debemos armarnos.

Le citoyen du monde n'est pas l'homme apatride ou acosmique, le voyageur universel qui se moque des frontières comme de la possession (ou de la privation) de droits garantis par un État. (...) Il est un être public, attentif aux expériences publiques de liberté dans le monde, et sa conscience cosmopolite s'intensifie et s'élargit lorsqu'elle prend part, voire prend parti, aux expériences républicaines dans le monde (Kant, 2006, p. 38).

La hospitalidad en sí no está exenta de hostilidad, y los medios que utiliza para incorporar al extranjero son las mismas medidas para neutralizarlo. La misma ambivalencia de la hospitalidad, que debe proporcionar garantías y seguridad para el extranjero y su anfitrión, puede dar a su reverso, la inhospitalidad, un sorprendente parecido. Gotman (2001) dijo que dar la bienvenida pero dejar de devolver es la filosofía del lugar que también es exactamente la de la hospitalidad. Entre estas diferentes trampas, la hospitalidad urbana es un camino estrecho. La hospitalidad se inventa, se organiza, pero también se le debe dar rienda suelta. El sistema de hospitalidad tiene capacidades limitadas, es necesariamente selectivo; no siempre puede conciliar los imperativos de igualdad y pluralidad.

La ausencia de republicanismo y de un derecho cosmopolita del Estado ha llevado a condiciones inhumanas para el refugiado en la escena contemporánea, incluso si el estatus que le otorgan las convenciones internacionales le garantiza los derechos. Más allá de estas especificidades de los refugiados tratados anteriormente, existen las perspectivas de inmigración debido a la cuestión ambiental, cuyos actores son conocidos como refugiados ambientales, además de los diversos casos de retorno del país de origen. Aunque tal orden regulatorio es un hito para los tratados de hospitalidad y derecho internacional, los Estados los transmiten de acuerdo con sus intereses. Los deberes absolutos de respeto por los demás son suprimidos por la necesidad económica. Por lo tanto, los ideales de hospitalidad incondicional con un tratado de paz universal terminan yendo en la dirección opuesta. 


\section{Hospitalidad doméstica y pública: una dimensión política}

Nuestra modernidad ha despojado a la hospitalidad de su positividad. Ella ha llevado su profundidad y aura al extranjero, pensamiento en Brugère y Le Blanc (2017), sobre la escena contemporánea. A través del tema del fin de la hospitalidad, redescubrimos la idea de la corrupción de la moral entre los modernos. La modernidad más reciente ha amplificado este credo moral del fin de la hospitalidad a un credo político. La modernidad está desencantada y de la hospitalidad es uno de sus tópicos. La división de migrantes / refugiados es una construcción política destinada a clasificar la biopolítica de las vidas a rescatar, sancionando la observación que se pretende sin apelación pero que en realidad es una construcción según la cual el migrante económico no es el refugiado político. Está bien la retirada de la hospitalidad como un valor político, es como escuchar un grito de ayuda.

Para Boudou (2017), la hospitalidad, porque es política, nunca es un concepto puro. La hospitalidad es un asunto político que moviliza discursos e ideales que no son del todo obvios. La idea de la hospitalidad no puede ser completamente disipada por sus metáforas, su historia antigua y religiosa, o incluso la hipocresía colonial que podría justificar.

A pesar de esto, es de conocimiento común que los ideales nostálgicos de la antigua hospitalidad se han convertido en modernidad por prácticas utilitarias y económicas. En cualquier caso, las premisas sociológicas y filosóficas de la concepción de la hospitalidad parten de una estructura humana que proporciona luz a la cientificidad del campo. Sin embargo, la hospitalidad en sí misma terminó abarcando el campo comercial para tener otros espacios en la academia tomando en cuenta el entorno profesional, más precisamente en el caso del turismo.

$\mathrm{Al}$ abordar el significado conceptual de la hospitalidad, Camargo (2004) dimensionó los siguientes espacios del eje social para su estudio en Brasil: la hospitalidad doméstica, llamada así por el acto de recibir en el hogar que implica una mayor complejidad con respecto a los ritos; hospitalidad pública, considerada en el derecho de ir y venir, esto se incluye en la vida cotidiana de la vida urbana; La hospitalidad comercial, destacada en las estructuras comerciales de acuerdo con el surgimiento del turismo moderno y adaptada a la designación habitual de hotelería y restauración, y la hospitalidad virtual, concebida como consecuencia de la relación entre los usuarios de Internet.

A pesar de la importante delimitación didáctica propuesta por Camargo (2004) para los estudios brasileños, se hace difícil aceptar la hospitalidad comercial sin restricciones como un acto de dar, y menos aún como una acción incondicional por parte del otro. Gotman (2001, p. 10) considera que "le tourisme et le mouvement autonome des voyages, qui transforment des flux massifs d'invités payants et des populations entières en hôtes professionnels, trouvent auprès de l'État appuis et garanties, c'est qu'ici l'apport monétaire compense significativement l'épreuve de l'hospitalité".

La lógica del dinero en la llamada hospitalidad comercial pervierte las relaciones de donación porque lo convierte en un subterfugio para disfrazar el sistema comercial. Además, la hospitalidad es una relación social codificada y extraordinariamente limitada, sujeta a obligaciones precisas y formalizadas, y que, si resultan de la economía del don y la reciprocidad, también están sujetos a una condición fundamental, dígase la asimetría (Gotman, 2001).

La presuposición sociológica de la asimetría de la hospitalidad incluso subvierte las relaciones igualitarias en el marco de las relaciones sociales esperadas en el estado de derecho. En nuestra opinión, la función fundamental de la hospitalidad es promover un proceso de intercambio asimétrico, material o simbólico entre quienes brindan hospitalidad (los anfitriones) y quienes lo reciben (los invitados) con una aspiración incondicional a un convenio colectivo de paz. Esta predisposición a la hospitalidad se opone a las reglas comerciales del mercado y las reglas contractuales del Estado, donde estas últimas tienen la intención de cumplir con las primeras.

Los fenómenos migratorios del trabajo, el turismo, así como el movimiento autónomo de viajes, plantean a los Estados democráticos problemas de apertura y cierre, creando conflictos $\mathrm{y}$ desequilibrios (ver figura 01). Si el turismo compra sus derechos de visita, los viajes de trabajo implican el desarrollo de mecanismos legislativos de filtrado y control para la adquisición de los derechos de entrada, estadía e instalación que están en contradicción con el derecho de libre acceso y circulación que dichos Estados reclaman (Gotman, 1997). 
Al dimensionar la hospitalidad en el espacio, es importante situar la noción de Costa y Moesch (2017, p. 155) cuando afirman que el territorio es representado por e representante de diferentes escalas, como un hecho y como un fenómeno. De hecho, el territorio tiene lugar en los grupos humanos más diversos. En la perspectiva de los autores, el territorio sufre transformaciones y reinterpretaciones que cambian su escala, que se cataliza de una acción comunitaria situada a una operación militar o económica expresiva resultante de intereses multinacionales, convirtiéndose en un representante de acciones endógenas y exógenas, escalas interactivas y conexiones.

La hospitalidad también puede tener una dimensión territorial colectiva y un carácter de obligación que, durante mucho tiempo, fueron religiosos (y asociados con la idea de la caridad) y que hoy serían más una cuestión de servicio público y dominio de protección social (como viviendas sociales, hospitales, etc.), o en el campo comercial (hoteles en particular). Por último, sería la base de los derechos: derechos internos en los Estados que rigen la situación de las personas extranjeras, desplazadas y expulsadas (derecho de entrada, derecho de asilo); y convenciones interestatales que definen el marco de las relaciones diplomáticas. "L'hospitalité, définie comme ce qui permet à des individus, des familles de lieux différents (à des villes et des États également) de se faire société, se loger et se rendre des sevices mutuellement et réciproquement, est une question tout à la fois d'actualité et très ancienne" (Gotman, 1997, p. 5).

En estos modelos teóricos, la hospitalidad está vinculada a la praxis de lo social con respecto a sus derechos, más precisamente en el caso de los sujetos de esta investigación: los inmigrantes y su universo de alteridad. Bajo estos supuestos, la hospitalidad pública y doméstica asumiría una dimensión política significativa en la escena contemporánea para la recepción de grupos vulnerables. La hospitalidad es un modelo operativo cuyas características estructurales son identificables y cuyas variaciones son muy variables (especialmente cuando se pasa de la hospitalidad privada a la hospitalidad pública). La principal diferencia entre la hospitalidad doméstica y la pública es el lugar dado a los protagonistas de la interacción.

L'bospitalité publique et bureaucratique dont il sera question ici, et qui représente une avancée en soi, a en effet son revers en ce qu'elle substitue des mécanismes neutres du point de vue des liens sociaux aux rapports intersubjectifs engagés dans l'exercice de l'hospitalité privée. Une institution ne reçoit pas, elle exige au contraire de ses membres retrait et dégagement. Elle ne s'accomplit réellement que lorsqu'elle réussit à créer en son sein une entité d'accueil - service ou bureau chargée d'établir le contact mais également de faire écran entre l'accueillant et l'accueilli (Gotman, 2004, p. 202).

Figura 01 - Inmigrante en el comercio informal de souvenirs cerca del Museo del Louvre en París

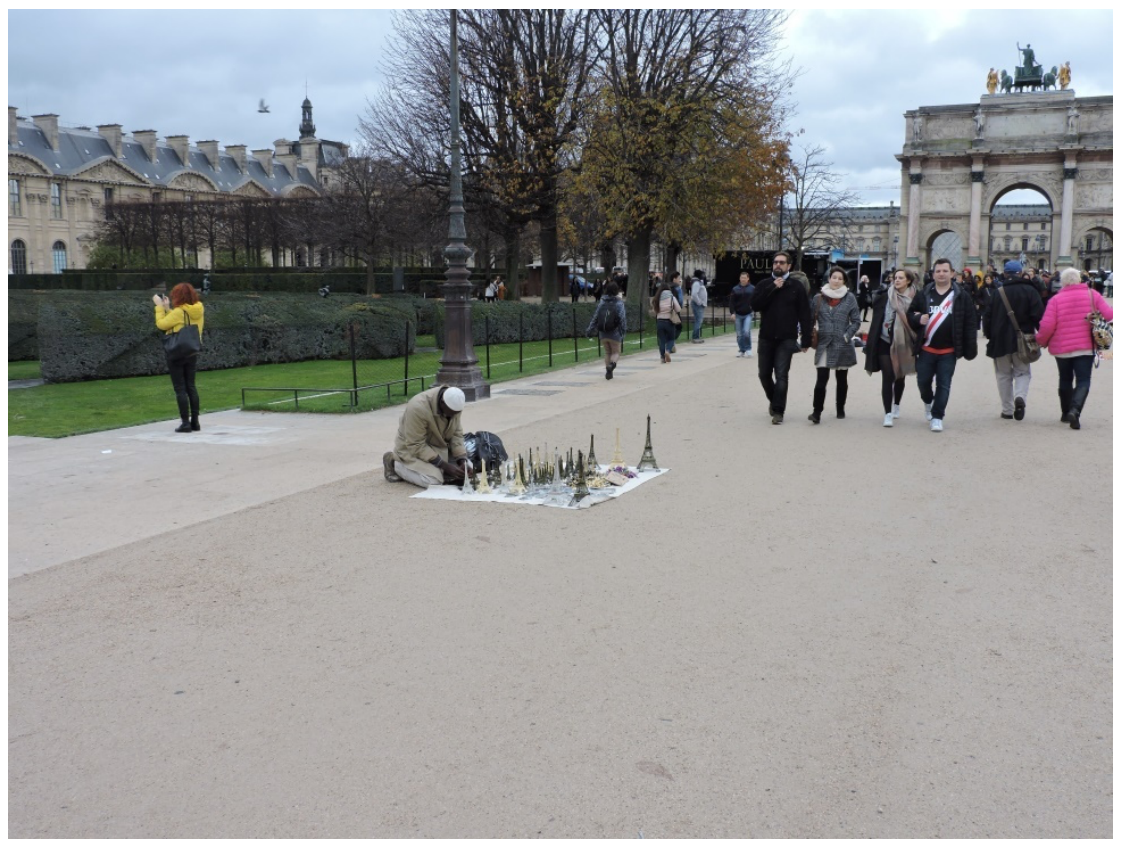

Fuente: Investigación del autor, diciembre de 2018 
La hospitalidad doméstica disminuirá a favor de la hospitalidad comercial, mientras que la hospitalidad pública será asimilada gradualmente por el sector filantrópico y finalmente por el Estado, porque la hospitalidad se ha transformado en derechos sociales y sistemas sociales. Sobre la solidaridad Gotman (2001, p. 605) se pregunta "L'hospitalité privée aurait-elle donc véritablement pris fin ? Oui, si l'on considère que l'hospitalité s'adresse aux inconnus et aux étrangers à la famille, ainsi que le veut le sens commun attribué à ce terme, auquel on préfère ceux, plus neutres, d'accueil ou d'hébergement".

La separación entre la hospitalidad doméstica y privada nos ha llevado a determinar que las funciones del Estado no se extienden a los ciudadanos como miembros del mismo y, en el sentido opuesto, el Estado delega a otras organizaciones las funciones que serían suyas. Esto crea un vacío demarcado para las acciones de hospitalidad política.

¿Por qué, entonces, la hospitalidad actual si rechazamos tanto la tesis del declive como la del lugar residual de la hospitalidad doméstica? La franca separación entre los agentes del Estado, los profesionales de la hospitalidad pública y la hospitalidad privada es precisamente el problema. La hospitalidad responde a una necesidad equivalente a la exogamia: la necesidad de aliarse con el exterior (Gotman, 2001).

La hospitalidad privada, anteriormente realizada como una forma de cohesión social en una comunidad dada, históricamente ha sido reemplazada por la hospitalidad pública contratada por el Estado a otras organizaciones. Según los modelos de la sociedad capitalista, el Estado delega estratégicamente este papel en organizaciones filantrópicas, religiosas o no gubernamentales porque se entiende como algo inferior en comparación con la gestión financiera de su capital-territorio. De esta forma, la hospitalidad pública no se considera como una plataforma política del Estado y, cuando se inserta allí, se reverbera a la inversa: las políticas de hostilidad en la gestión de sus fronteras. Ante esta separación entre la hospitalidad doméstica y la pública, el Estado termina subcontratando acciones de hospitalidad con respecto a la solidaridad, pero mantiene para sí acciones de control migratorio dirigidas a austeridad y hostilidad hacia el extraño que no quiere como miembro.

La hospitalidad se basa en el principio de asimetría, aunque este puede ser el límite o incluso la negación. Es esta regla de asimetría la que distingue la hospitalidad del contrato y hace posible evitar la instalación; lo que hace que la hospitalidad sea un don. Esto es cierto para las reglas de entrada tanto en la esfera privada como en la esfera pública. "Hier obligation collective, elle est aujourd'hui laissée à l'initiative de particuliers. (...) L'hospitalité est passée aujourd'hui de la sphère religieuse et privée à la sphère étatique, dans le droit (droit social, droit international) et s'exerce par voie de redistribution" (Gotman, 2001, p. 14). Estas diferentes tradiciones han sido reforzadas por la idea de lo sagrado de la hospitalidad construida, en primer lugar, por la asimilación del extranjero a Dios.

\section{4. ¿El fin de la hospitalidad?}

¿Podemos por lo tanto trazar una política de hospitalidad para el mundo contemporáneo? En lo que respecta al cosmopolitismo kantiano, la ciudadanía europea parece ser el legado más exitoso; Los artículos 13 (derecho a emigrar), 14 (derecho a asilo) y 15 (derecho a la nacionalidad) de la Declaración Universal de Derechos Humanos cerrarían la brecha entre las preocupaciones sobre la producción en masa de apátridas y refugiados de los siglos XX y XXI.

La inmigración, de hecho, no está exenta de consecuencias para el espacio doméstico. Cuando el espacio privado se "vende" a los internos que lo invaden, al tiempo que proporciona recursos adicionales preciosos y, para los inmigrantes más religiosos, profana la santidad del hogar, la familia se desorganiza. Es en el mantenimiento de este desequilibrio que reside, como hemos ya visto, una de las condiciones de realización de a hospitalidad. Un desequilibrio perteneciente a la economía del don. La hospitalidad opera a la inversa, convierte al enemigo en un anfitrión.

Según Gotman (2001, p. 149) "accueillir l'hôte ‘à bras ouverts' n'est pas à proprement parler codifiable dans des règles d'étiquette mais laissé au contraire à l'appréciation de celui qui donne (l'hospitalité), dès lors exposé au risque de ne jamais donner assez". Regresamos a Derrida (1997) cuando dijo que sólo la hospitalidad justa rompe con la hospitalidad jurídica; no es que la condene o se oponga, por el contrario, puede expresarla y mantenerla en un movimiento incesante de progreso. Es la inversión de la hospitalidad en la hostilidad a partir de la perversión siempre posible de la ley. Esto nos lleva a pensar en la lengua 
materna como una metáfora del hogar en el otro, un lugar sin lugar que se abre al significado de la hospitalidad.

Issue de la séparation des membres et des non-membres, des citoyens à part entière et des citoyens de seconde zone, l'hospitalité ne concernerait-elle que les paurres, démunis, sans domicile et, si oui, n'est-il pas imprudent de convoquer un terme qui évoque une logique caritative et une tradition chrétienne auxquelles les politiques publiques sont censées substituer une logique égalitaire d'accès aux droits? (Gotman, 2003, p. 12)

El hecho es que la globalización excesiva y las regulaciones estatales proteccionistas hacen que la hospitalidad para otros sea difícil y peligrosa, esencialmente en la lógica de la inmigración contemporánea. Con respecto al papel del Estado, se reconoce que su importancia en la migración internacional no significa que sea necesariamente el factor más relevante en la formación y mantenimiento de los flujos. La migración internacional no es causada exclusiva o principalmente por la acción estatal. Sin embargo, gracias a las políticas de inmigración y ciudadanía, resulta un factor explicativo importante en el proceso de formación de flujos y ayuda a moldear la forma que adquieren estos flujos (Reis, 2006).

En cualquier caso, la participación del Estado es de suma importancia en la resolución de conflictos resultantes de estos flujos migratorios. $\mathrm{Al}$ señalar que existe intolerancia y que está arraigada en la cultura de las personas de acogida, el Estado debe asumir la responsabilidad de una acción política proactiva en el sentido de desarrollar condiciones prácticas favorables para la recepción de refugiados e inmigrantes. Los flujos migratorios colocan a las personas en las más diversas situaciones de vulnerabilidad al ingresar a un nuevo país con otro orden normativo social y económico.

L'État, comme la famille, tend à réduire les ouvertures, qui sont dès lors périodiquement à rouvrir, par la force, par l'infraction aux règles, à la marge des institutions, ou en les détournant. L'hospitalité, en ce sens, n'est pas un état mais un processus permanent de réouverture et de relance : recommandations, passe-droits viennent alors corriger (mais aussi subvertir) des critères de sélection trop restrictifs et réintroduire de la pluralité dans la redistribution des biens (Gotman, 1997, p. 15).

Depende del propio Estado promover y asegurar la paz tanto interna como externa. Los Estados Armados para la Guerra deben consultar las máximas de los filósofos sobre las condiciones de posibilidad de la paz pública. Una doctrina universal de la prudencia puede coexistir con la moral, pero no con un moralismo político que forje una moral que sea beneficiosa para el interés del estadista. Si es un deber, si hay al mismo tiempo una esperanza bien fundada de realizar el estado de un derecho público, incluso si uno solo puede abordarlo mediante un progreso indefinido, la paz perpetua, que resulta de lo que hasta ahora hemos llamado erróneamente tratados de paz no es una idea hueca, sino un problema que se resuelve gradualmente y se acerca constantemente a su objetivo: alianzas de paz que son necesariamente cosmopolitas (Kant, 2006).

Los municipios tienen y utilizan diferentes medios para elegir su población y seleccionar a sus nacionales. Las fronteras de la hospitalidad no son sólo nacionales, sino municipales $\mathrm{y}$, en general, administrativas. Tanto la hospitalidad como la ley se basan en la definición de besoin en cuyo nombre el outsider será elegible para su protección (internacional, nacional, municipal o nacional), en las condiciones precarias que nos legitiman para ayudar.

Por lo tanto, fue bajo el efecto de la división del trabajo social y el avance de la ley que no dejó de continuar y recomponer que la protección de los pobres, a primera vista religiosos y municipales, pasó gradualmente a las manos. filantropía y el Estado, y que los extranjeros han sido puestos bajo la protección de convenciones bilaterales y luego internacionales. Sin embargo, estas categorías no son fijas ni estancas; y son testigos del mantenimiento y la renovación de la hospitalidad religiosa comunitaria o del desarrollo de formas mixtas de hospitalidad colectiva (Gotman, 2001).

El extraño puede ser experimentado de diferentes maneras, como la diferencia (para comprender), la anomalía (para corregir), el peligro (para eliminar), el recién llegado (para iniciar), el enemigo (para luchar), el viajero (para proteger), el invitado (para dar la bienvenida), más generalmente el tabú (para evitar o controlar), el misterio (para desmitificar o respetar), lo extraño (para familiarizarse), etc. (Boudou, 2017). Integradas con las políticas de migración, las políticas de integración también dependen de las historias nacionales.

Para Bauman (2004), los extranjeros no son un invento moderno, pero aquellos que siguen siendo extranjeros durante mucho tiempo, o incluso perpetuamente, lo son, pues en una ciudad 
o en un pueblo premoderno, no se le permitió seguir siendo extranjero durante mucho tiempo. Algunas construcciones urbanas modernas sirven para segregar y excluir, y no para construir puentes, pasajes accesibles y puntos de encuentro, para facilitar la comunicación o, de alguna otra manera, para acercar a los habitantes de la ciudad.

Si la ciudad, desde su origen, constituye una vivienda colectiva, dar, recibir y devolver no puede dejar de estar en el corazón de su territorio. Desde un punto de vista económico, el territorio está estructurado de manera fragmentada y su vínculo más amplio reside en la búsqueda de una compensación colectiva y la difusión del obsequio. Uno de los múltiples poderes que emanan del territorio es el don en sí mismo en su gratuidad. El territorio debe ser asumido en construcción, en comunión (Costa \& Moesch, 2017).

El extranjero no puede reclamar un derecho de residencia, sino un derecho de visita: este derecho, dado a todos los hombres, es el de ofrecerse a la sociedad, en virtud del derecho de posesión común de la superficie de la Tierra; como ésta es esférica, no pueden dispersarse en ella hasta el infinito, sino que deben apoyarse mutuamente. Originalmente, nadie tiene más derecho que otro para ocupar dicho lugar. La inhospitalidad de las costas marítimas, que permite piratear barcos en los mares vecinos, es, por lo tanto, contraria al derecho natural, pero el derecho de hospitalidad, es decir, la autorización otorgada a los arribos extranjeros, se detiene al buscar las condiciones de posibilidad de un comercio con los antiguos habitantes (Kant, 2006).

La hospitalidad y la inhospitalidad pueden, por lo tanto, adoptar lenguajes muy similares. Si la inhospitalidad es una dimensión pragmática de la expulsión de los judíos de Francia de 1941 a 1944, la expulsión de la ciudadanía, el imperio de la ley, la ciudad económica y, finalmente, del territorio, es que un país convirtió parte de sus miembros en no miembros para entregarlos al enemigo. Un funcionario del gobierno argumenta que "abuso de nuestra hospitalidad", argumento utilizado convencionalmente contra todos los extranjeros a los que el régimen se está preparando para internar o expulsar, aborda Gotman (2001) sobre la cuestión política de colaboración y responsabilidad. Autoridades francesas en la deportación de judíos durante la Segunda Guerra Mundial.

Hoy, una reflexión sobre la hospitalidad presupone, entre otras cosas, la posibilidad de una delimitación rigurosa de umbrales o fronteras: entre la familia y la no familia, entre el extranjero y el no extranjero, el ciudadano y el no -ciudadano, pero primero entre lo privado y lo público, derecho privado y público, etc.

L'hospitalité peut, à bien des égards, être considérée comme un rituel d'exploration. L'bospitalité n'a en effet rien de spontané, c'est une 'vertu', disent les anciens, une pratique culturelle dirions-nous aujourd'bui, c'est en quelque sorte un pré-droit qui vise à intégrer temporairement l'étranger qui franchit le seuil de la maison, à lui ménager une place honorable sans pour autant que l'hôte perde sa souveraineté, et à suspendre les conflits d'appartenance et d'inégalité de condition qui subsistent entre un hôte qui est chez lui ('chez' = casa, maison) et un étranger qui, lui, est tempoairement dépourvu de maison (Gotman, 2013, p. 13).

En esta dirección, la apertura a los intercambios asimétricos y sus donaciones se relega a pocas instituciones o grupos sociales locales que, incluso si aspiran a la incondicionalidad y a un tratado de paz, terminan siendo rehenes de descuido del estado o su opresión. "En tempos de crise, les villes prennent des mesures draconiennes d'expulsion", dice Gotman (2013, p. 8), un hecho que subraya la hospitalidad al servicio de la industria se muestra cuando se dice que la actitud de las ciudades era casi igual en todas partes: en tiempos de crisis, se tratara de una hambruna, una amenaza de asedio, una epidemia o tensiones sociales graves, se tomaban medidas draconianas para expulsar a los pobres (Zeller, 2004).

En el contexto del migrante refugiado, Pereira (2016, p. 1) se pregunta: “Qu'en est-il de l'application du concept d'hospitalité quand l'être humain doit fuir son pays d'origine en raison d'une crainte de persécution par inefficacité de la protection de l'État ? Ou, pire encore, quand le persécuteur est l'État lui-même ?".

Es un hecho que, durante los dos siglos de la historia moderna, las personas que no han logrado convertirse en ciudadanos, refugiados, migrantes voluntarios e involuntarios, personas desplazadas por completo, han sido consideradas naturalmente como un problema de país anfitrión y tratado como tal. Quizás sería mejor cambiar las costumbres del mundo y hacer que nuestro hábitat sea más hospitalario hacia la dignidad humana, para que madurar no requiera el compromiso de la humanidad de un niño. El valor humano más preciado es el atributo sine qua non de la humanidad, no la supervivencia a toda costa.

Según Costa y Moesch (2017), el territorio como un hecho y como un fenómeno social es una prueba indiscutible de cruzar, errar, de la lucha 
permanente por "estar" en el mundo; territorio que exige y emana de la tríada de economía, cultura y poder, pero que también es compatible con la hospitalidad gratuita.

El mundo líquido, postulado por Bauman (2004), traduce parte de nuestras impresiones cuando dice que la fraternidad se ha convertido en una empatía para un pequeño grupo de personas y que los demás han sido rechazados porque no pertenecen a este círculo social. Los extranjeros y los extraños se convierten así en criaturas para repeler. Observado a través de la lente de un mundo ordenado, bien construido y en funcionamiento en armonía, la "zone grise de la solidarité", de amistad y asociaciones humanas es vista como el reino de la anarquía. "La question de l'hospitalité refait surface chaque fois que survient une crise, crise des sans-papiers ou 'crise des réfugiés' comme on l'appelle maintenant, qui suscite des prises de position politiques radicalisées en faveur de l'accueil ou, au contraire, en faveur du droit de décider d'accueillir ou pas" (Gotman, 2017, p. 3).

Lo que distingue la inmigración contemporánea de las migraciones anteriores es la importancia de los medios legales y administrativos del Estado en la vida cotidiana: la llegada al territorio nacional es, ante todo, una confrontación con las autoridades administrativas, para estancias sostenibles, anfitriones mal dotados; sin embargo, organizados. Éstos, por su parte, tienen el comité que recibirá una imagen igualmente precisa (si no justificada) que se utilizará para acercarse al extranjero. Además de la injusticia de los criterios de admisión, hay bloqueos de comunicación.

"L'hospitalité intéresse ainsi la politique urbaine du point de vue des déplacements, des circulations et des migrations, qui forment plus que jamais l'horizon dans lequel villes, États et citadins coexistent, et en fonction duquel les différents types de services s'organisent, de façon plus ou moins efficace" (Gotman, 1997, p. 9).

Hoy en día, la hospitalidad pública hacia el extranjero se caracteriza por su ausencia con la expulsión de inmigrantes y el rechazo por parte del Estado (Figura 02). El fin de la hospitalidad estatal es $\sin$ duda el veredicto que traemos de Calais (Francia), pero no significa el fin de la hospitalidad para individuos o grupos. La hospitalidad nunca termina, ¿cómo llegamos a esta negación de hospitalidad $O$, peor aún, a este crimen de hospitalidad? (Brugère \& Le Blanc, 2017)

Figura 02 - Evacuación de campamentos de migrantes en Porte de la Chapelle en París

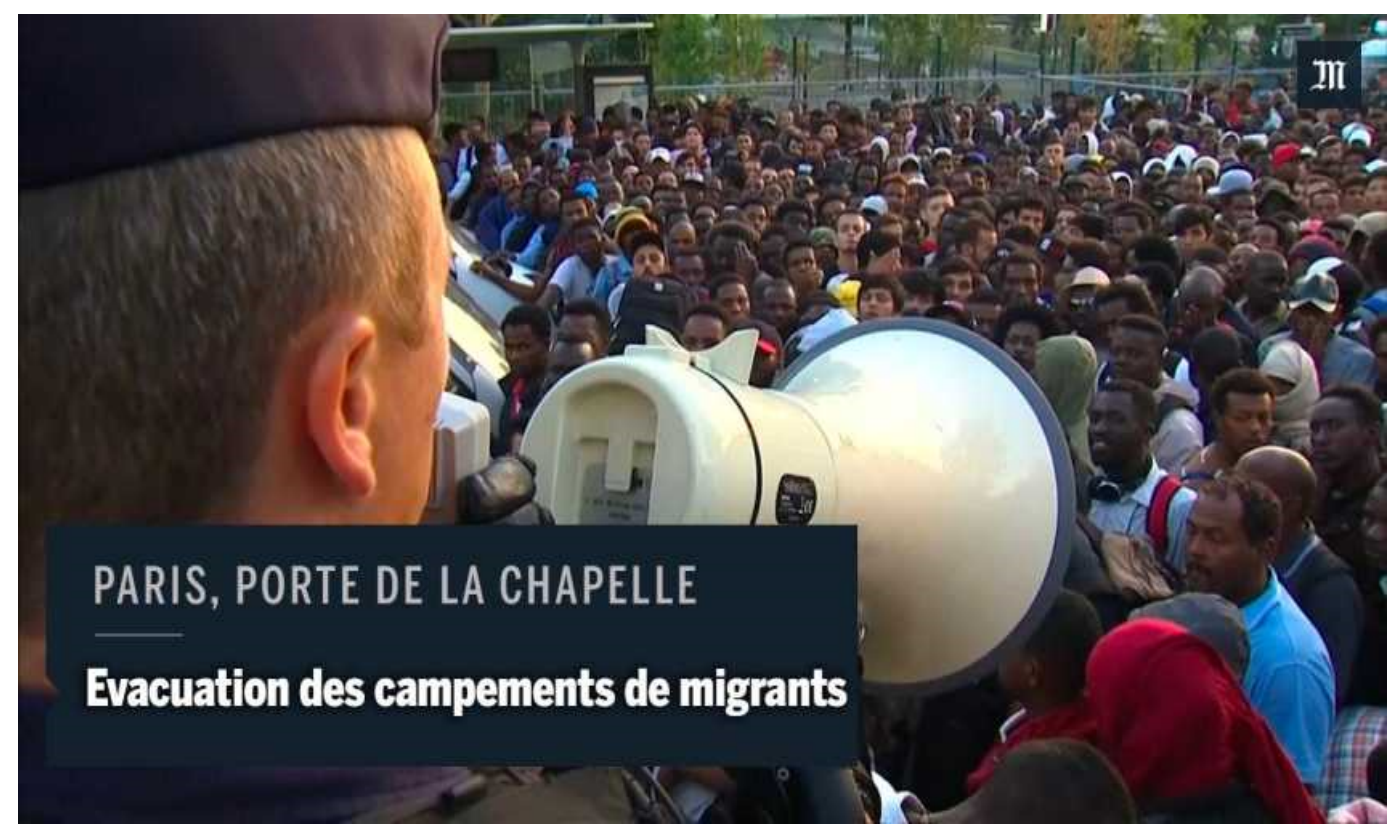

Fuente: https://www.lemonde.fr/societe/video/2017/07/07/paris-evacuation-des-migrants-installes-porte-de-lachapelle 5157259 3224.html 
Así, en nuestra opinión, las denominaciones emblemáticas "terre d'asile" en Francia: es una apariencia que se aparta de la esencia de las relaciones humanas con respecto a las relaciones fenomenológicas en el tratamiento público de migrando con muchas puertas y muros para cruzar frente a la modernidad. Es la distopía de la hospitalidad contemporánea.

\section{Conclusión - une crisis de la hospitalidad}

Los métodos de recepción deben tener como objetivo la hospitalidad asociativa para integrar a los sujetos migrantes en los diversos sectores de la sociedad y sus actividades, como el trabajo, el ocio, la educación, la cultura, etc. Sin embargo, sobre la base de los cargos legales, la reciente criminalización de organizaciones de este tipo, listas para llevar a cabo este trabajo, refuerza nuestra crisis de hospitalidad. El viejo concepto de hospitalidad fue suprimido por un supuesto anacronismo frente a las características de la modernidad líquida y de la sociedad de individuos. En el discurso nacionalista, el extranjero se inserta como una invasión del territorio: el mismo espacio que anteriormente formaban otras generaciones de inmigrantes. Por lo tanto, la obligación de acoger al otro como una acción de cosmopolitismo moral y una actitud altruista naufraga en la contemporaneidad. El término crisis migratoria difundido por los medios contemporáneos y por funcionarios estatales es, ante todo, una crisis de hospitalidad.

Partimos de una premisa del territorio como uno de los lugares del don y de las relaciones de alteridad con el otro, ya que es un núcleo indiscutible de la vida de las relaciones, con sus condiciones particulares de reproducción social y guardián de los significados existenciales. Desde formas de vida ubicadas en una relación total de la sociedad que conforma el mundo [el sentido mismo de la humanidad], todos revelan los personajes que producen el territorio a través de los usos y las apropiaciones, es decir, la forma de tomar el camino de la apropiación territorial. Acusa a las dimensiones reales de dar, de recibir (Costa \& Moesch, 2017). Dichos requisitos son condiciones sine qua non para prácticas de hospitalidad.

Así, a pesar de las propuestas de recibir inmigrantes y refugiados de ciertas instituciones desde un punto de vista territorial, que continúan infundiendo hospitalidad en su praxis de intercambios asimétricos, las escenas de hospitalidad imaginadas por este investigador se remontan al purgatorio medieval con el tortura y oscuridad. Le Goff (1981, p. 436) ya se había advertido que "le Purgatoire apparaît aussi dans les principales manifestations des nouvelles formes de solidarité entre les vivants et les morts au XIII siècle". La elección entre el Infierno o el Paraíso, ya que el Purgatorio era la antecámara asegurada del Paraíso, todavía se podía decidirse en el último minuto. Los últimos momentos fueron los de la última oportunidad. Queda por ver si los inmigrantes aún tendrán una oportunidad en el purgatorio contemporáneo entre el infierno del que creían que huían de su país de origen y el paraíso que estaban buscando en sus destinos inciertos.

Las políticas de hospitalidad deberían hacer propuestas para acoger al otro en su imposibilidad por medio de intercambios asimétricos con aspiraciones incondicionales. Sin embargo, el estado contemporáneo incorpora su nacionalismo y toma el camino opuesto del derecho al cosmopolitismo y la paz. Él mismo transfiere la responsabilidad de la solidaridad a las instituciones no gubernamentales y religiosas, pero mantiene el control sobre asuntos legales y policiales en sus fronteras y territorios. El discurso proteccionista del estado oculta la hostilidad hacia los no miembros y la xenofobia migratoria, así como contra el turismo para una parte de su población. Por el momento, todavía tenemos que identificar las propuestas de los individuos y la mercantilización de las personas. Por lo tanto, buscamos pensar en la praxis diaria de los intercambios sociales con los demás, los extranjeros, ya que queremos pensar académicamente sobre cómo (re)construir vínculos sociales en la modernidad.

\section{Referencias bibliográficas}

Bauman, Z. (2004). Amor líquido. Sobre a fragilidade dos laços humanos. Rio de Janeiro: Zahar.

Boudou, B. (2017). Politique de l'hospitalité. Paris: CNRS Éditions.

Brugère, F., \& Le Blanc, G. (2017). La fin de L'bospitalité. Paris: Flammarion.

Caillé, A. (2002). Antropologia do dom: terceiro paradigma. Petrópolis, RJ: Vozes.

Camargo, L. O. L. (2004). Os domínios da hospitalidade. In: A. F. M. Dencker \& M. S. Bueno (Orgs.). Hospitalidade: cenários e oportunidades. São Paulo: Pioneira Thomson Learning. 
Costa, E. B. da, \& Moesch, M. M. (2017). Território - locus da dádiva e núcleo da vida In: L. B. Brusadin (Org.). Hospitalidade e Dádiva: a alma dos lugares e a cultura do acolhimento. Curitiba: Prismas.

Derrida, J., \& Dufourmantelle, A. (1997). De l'hospitalité. Paris: Calmann-Lévy.

Durkheim, E. (1960). De la division du travail social. Paris: PUF.

Godbout, J. T. (1992). O espírito da dádiva. Paris: Éditions La Découverte.

Gotman, A. (1997). La question de l'hospitalité aujourd'hui. Communications, 65, L'hospitalité, pp. 5-19. Recuperado em 14 de novembro de 2018, https://www.perse e.fr/doc/comm 058880181997 num 651 1983?\&sa=u\&ei= 8pq3volihztfatakgfab\&ved $=0$ ccyqfjad\&usg 三afqjenhjz3nscjeecjepips $4 \mathrm{~m} 2$ w80buw.

Gotman, A. (2001). Le sens de l'hospitalité. Essais sur les fondements sociaux de l'accueil de l'autre. Paris: Presses Universitaires de France.

Gotman, A. (2003). Barrières urbaines, politiques publiques et usages de l'hospitalité. Les annales de la recherche urbaine, n. 94, L'accueil dans la ville, 6-15. Recuperado em 14 de novembro de 2018, http://www.annalesde larechercheurbaine.fr/IMG/pdf/Gotman ARU 94.pdf

Gotman, A. (org.). (2004). Villes et hospitalité. Les municipalités et leurs "étrangers». Paris: Éditions de la Maison des sciences de l'homme.

Gotman, A. (2013, octobre). Du Capitaine Cook à l'hôte administratif: l'hospitalité au sens propre et au sens figuré. In Santé et politiques d'accueil, Bordeaux, France.

Gotman, A. (2017). L'hospitalité est-elle soluble dans le droit ?. In: M. Wieviorka (Ed.). Les Solidarités. Auxerre, France: Éditions Sciences Humaines.

Kant, E. (2006). Ver la paix perpétuelle. Flammarion: Paris.

Le Goff, J. (1981). La naissance du purgatoire. France: Éditions Gallimard.

Mauss, M. (2008). Ensaio sobre a dádiva. Lisboa: Edições 70.

Montandon, A. (2002). Ritos da hospitalidade erótica. In: C. M. de M. Dias (Org.). Hospitalidade: reflexões e perspectivas. São Paulo: Manole.

Pereira, J. C. (2016). Acolhida a migrantes e refugiados: a ética da pastoral do migrante e desafios para a democracia no Brasil. Travessia - revista do migrante, ano XXIX, $\mathrm{n}$. 79, 101-126.
Reis, R. R. (2006). Soberania, direitos humanos e migrações internacionais. Revista Brasileira de Ciências Sociais, 19(55), 149-163. Recuperado em 20 de março de 2019, https://dx.doi.org/10.1590/S010269092004000200009 .

Spolon, A. P. G., \& Brusadin, L. B. (2016). O Brasil no cenário da pesquisa contemporânea internacional em hospitalidade: da oportunidade de rompimento de fronteiras e da criação de uma rede colaborativa de estudos e pesquisas. Revista Turismo e Desenvolvimento, n. 26, 47-64. Recuperado em 18 de março de 2018, http://revistas.ua.pt/index.php/r td/article/view/6155/4883.

Zeller, O. (2004). La place des miséreux et des malades à Lyon, de l'Ancien Régime à nos jours. In: A. Gotman (org.). Villes et hospitalité. Les municipalités et leurs «étrangers». Paris: Éditions de la Maison des sciences de l'homme. 